\title{
Seizures With Major Comorbidity and Complications: Association of the Teaching Status of the Hospitals With the Outcomes
}

\author{
Aparna Yarram ${ }^{\mathrm{a}}$, Ali Seifi ${ }^{\mathrm{b}}$, Vahid Eslami ${ }^{\mathrm{b}, \mathrm{c}}$
}

\begin{abstract}
Background: This study aims to compare the outcomes of complicated seizure cases in teaching institutions as compared to non-teaching hospitals.
\end{abstract}

Methods: A retrospective cohort study was conducted utilizing the Healthcare Cost and Utilization Project (HCUP) national database to analyze outcomes of seizures between 2012 and 2016 in the USA.

Results: We evaluated 267,430 of seizure patients with major complication or comorbidity between 2012 and 2016. Of these, 6,980 in-hospital deaths were reported. There was a trend toward a significantly higher mortality in teaching compared with nonteaching hospitals $(\mathrm{P}=0.07)$. The average length of stay (LOS) was 5.2 days, with LOS in 2014 and 2016, being longer in teaching hospitals $(\mathrm{P}<0.05)$. Hospital charges were not significantly different among the two groups, but both types of hospitals did show a statistically significant charge increase from 2012 to 2016 $(\mathrm{P}<0.001)$.

Conclusions: Our data showed that there is a trend toward significantly higher mortality in teaching hospitals. LOS was also more reported in teaching hospitals, which could be inherent to the increased volume and coordination of care and more complexity of the cases in teaching hospitals. However, hospital charges were not different in teaching versus non-teaching hospitals.

Keywords: Epilepsy; Teaching hospitals; Outcomes

Manuscript submitted May 20, 2020, accepted June 10, 2020

Published online June 17, 2020

aSchool of Medicine, University of Texas Health Science Center at San Antonio, San Antonio, TX, USA

bDepartment of Neurosurgery and Neurology, University of Texas Health Science Center at San Antonio, San Antonio, TX, USA

${ }^{\mathrm{c} C}$ Corresponding Author: Vahid Eslami, Department of Neurosurgery and Neurology, University of Texas Health Science Center, 7703 Floyd Curl Drive, San Antonio, TX 78229, USA. Email: Eslamiv@uthscsa.edu

doi: https://doi.org/10.14740/jnr588

\section{Introduction}

World Health Organization (WHO) estimates that up to $10 \%$ of the population worldwide will have at least one seizure during their lifetime [1]. Seizures account for $1-2 \%$ of all emergency department visits, and approximately $25 \%$ of these visits are noted to be a first-time seizure $[2,3]$. New-onset seizures may occur due to central nervous system (CNS) infections, tumors, chemical and metabolic imbalances, drugs, etc., and maybe a one-time event. However, seizures are most commonly part of a chronic condition characterized by recurrent seizure events known as epilepsy. Epilepsy affects 50 million people worldwide, and in $2015,1.2 \%$ of the USA population reported active epilepsy [4]. Epilepsy and seizures contribute to many comorbidities, including neuropsychiatric issues, and significantly impact the patient's quality of life [5]. Investigating what factors contribute to mortality in epilepsy patients will allow us to gain a better understanding of how to improve treatment and decrease hospitalizations for these patients.

Past studies comparing overall hospitalizations for all medical illnesses have shown that there is a significant decrease in mortality in major teaching hospitals compared to non-teaching hospitals [6]; however, complicated seizures, defined as seizure cases with major comorbidity and complications during their hospitalizations, have not been studied in depth in the literature. In order to better understand the differences amongst teaching and non-teaching hospitals, our study aims to compare the mortality, hospital length of stay (LOS) and hospital charges of complicated seizure cases with other comorbidities that are occurring in teaching institutions as compared to the non-teaching hospitals in the USA.

\section{Materials and Methods}

We conducted a retrospective cohort study utilizing the Healthcare Cost and Utilization Project network (HCUPnet) national database to compare mortality, LOS, and hospital charges of patients presenting with seizures in teaching vs. non-teaching hospitals between 2012 and 2016. HCUP is a national database and software tool sponsored by the Agency for Healthcare Research and Quality (AHRQ). The database represents the 
Table 1. Patient and Hospital Demographics Between Teaching and Non-Teaching Institutions

\begin{tabular}{lllll} 
& Mean in all cohorts & Teaching & Non-teaching & Relative ratio (T/NT) \\
\hline Number (\%) & 53,486 & $195,224(73)$ & $72,206(27)$ & 2.70 \\
Age (years) & 49.24 & 45.40 & 57.06 & 0.80 \\
LOS days & 5.20 & 5.30 & 5.02 & 1.06 \\
Hospital cost & $\$ 12,670$ & $\$ 13,383$ & $\$ 11,280$ & 1.19 \\
Hospital charge & $\$ 48,975$ & $\$ 50,382$ & $\$ 49,168$ & 1.02 \\
Mortality (\%) & $2.16 \%$ & $2.19 \%$ & $2.12 \%$ & 1.03 \\
\hline
\end{tabular}

T: teaching; NT: non-teaching; LOS: length of stay.

most extensive collection of longitudinal hospital care data in the USA, including the synthesis of data from state healthcare centers, hospital associations, private institutions, and the federal government.

We identified patients categorized under Medicare Severity Diagnosis-Related Groups (MS-DRGs) with the code number 100 for seizure cases with major comorbidity and complications. We compiled total values of discharges and compared the data against different categories such as in-hospital deaths, hospital LOS, and hospital charges. Trends during the 5 years of study were evaluated using the $\mathrm{z}$-test statistic and $\mathrm{P}$ values using a two-tail test with a significance of less than 0.05 . The HCUP is an anonymous database and fully complied with the ethical standards and received "exempt" status from the Institutional Review Board (HSC-2015-0408N).

\section{Results}

\section{Demographics}

We evaluated 267,430 of seizure patients with major complication or comorbidity between 2012 and 2016. The prevalence of the seizure discharges slightly increased from 60,825 in 2012 to 71,350 in 2016 with an average rate of 18.88 in 100,000 persons. The mean age of the total cohort had minimal variation and was 49.2 years. Male patients had a slightly younger age at presentation, with an average of 47.1 years, and female patients had an average age of 51.5 years. Male patients made up $52.2 \%$ of the discharges in this cohort (Table 1 ).

\section{In-hospital deaths}

The average number of in-hospital deaths for each of the 5 years analyzed was 1,396, with a total of 6,980 , and mean $2.16 \%$ deaths per discharges. The absolute number of inhospital deaths remained relatively consistent yearly, ranging between 1,315 and 1,530. The average percentage of deaths in teaching hospitals from 2012 to 2016 was $2.19 \%$, and in non-teaching hospitals was $2.12 \%$. There was a trend toward significant higher mortality in teaching compared with nonteaching hospitals $(\mathrm{P}=0.07)$ (Fig. 1).

\section{LOS}

The average LOS for the years examined was 5.2 days and ranged from 5.1 to 5.3 days. The average LOS in teaching hospitals was $5.3 \pm 0.5$ days, whereas the average LOS in non-teaching hospitals was 5.0 days. There was a significant increase in LOS in teaching hospitals compared with nonteaching hospitals in the years 2014 and 2016, P $<0.05$ (Fig. 2). In 2014, the mean LOS in teaching hospital was 5.31, while in the non-teaching hospital was $4.97(\mathrm{P}=0.02)$. In 2016, the LOS in teaching hospital was 5.40, and the average in the nonteaching hospital was $4.92(\mathrm{P}<0.001)$. The remainder of the

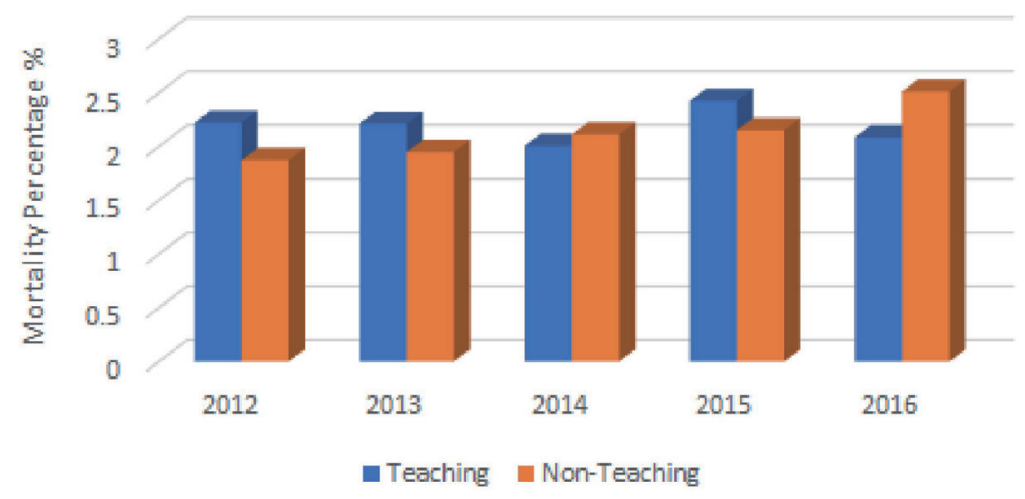

Figure 1. Trend of annual in-hospital deaths for seizures in teaching vs. non-teaching hospitals in USA from 2012 to 2016. 


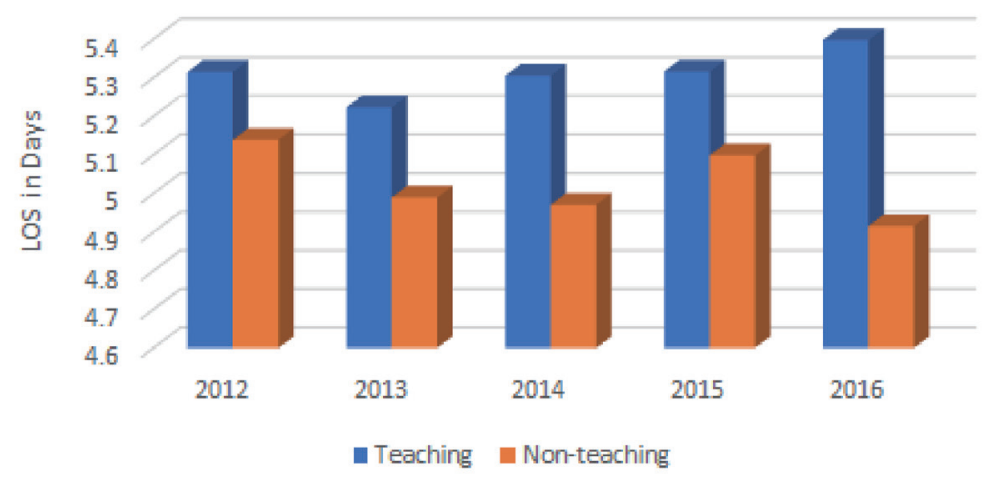

Figure 2. Trend of hospital LOS for seizures in teaching vs. non-teaching hospitals in USA from 2012 to 2016 . LOS: length of stay.

years was not significantly different in between the two types of hospitals (Fig. 2).

\section{Hospital charges}

The total average hospital charges for patients presented with seizures between 2012 and 2016 were \$48,975 and ranged from $\$ 43,704$ in 2012 to $\$ 55,500$ in 2016 . The average hospital charges in teaching hospitals were $\$ 50,382$, whereas, in non-teaching hospitals, the average was $\$ 49,169$ ( $\mathrm{P}>0.05$, Fig. 3). There was a significant increase in hospital charges in both teaching and non-teaching hospitals from 2012 to 2016. Teaching hospitals had an average hospital charge of $\$ 44,626$ in 2012 to $\$ 57,443$ in $2016(\mathrm{P}<0.001)$, and non-teaching hospitals had an average hospital charge of $\$ 45,442$ in 2012 to $\$ 54,228$ in $2016(\mathrm{P}<0.001)$.

\section{Discussion}

Our study examined outcomes of patients with seizures from 2012 to 2016 among teaching hospitals and non-teaching hospitals in the USA. Our findings showed that there is a trend toward significant higher mortality in teaching compared with non-teaching hospitals. In 2014 and 2016, there was a substantial increase in the LOS of seizure patients in teaching hospitals as compared to non-teaching hospitals. The costs of inpatient hospitalizations did not differ between the two groups of teaching and non-teaching hospitals. One explanation could be faster admission-to-discharge process, but more costly procedures in non-teaching hospitals. The other possibility is prolonged LOS in teaching hospitals, but as costly as non-teaching hospitals. Teaching hospitals are known for very specialized care and for treatment of uncommon or rare diseases [7]. However, for more frequent conditions, teaching hospitals may provide a lower care than non-teaching hospitals, because teaching hospitals may have a high contribution of inexperienced trainees and decreased role of senior physicians. This may end in more LOS, with higher costs seen in teaching hospitals [7]. Mortality of seizure and epilepsy-related deaths in hospitals has been shown to be decreasing in the last two decades [8]. We found higher mortality in teaching hospitals compared with non-teaching ones. No prior study was found in epilepsy. However, literature has conflicting reports regarding hospital mortality in teaching hospitals in other fields. For example, Hyder et al presented improved mortality of patients undergoing hepatopancreatobiliary surgeries in teaching hospitals [9]; and Fineberg et al demonstrated increased mortality and hospitalizations of patients undergoing cervical spine surgery in teaching hospitals [10]. However, it has been shown consistently that even in studies that observed higher mortality

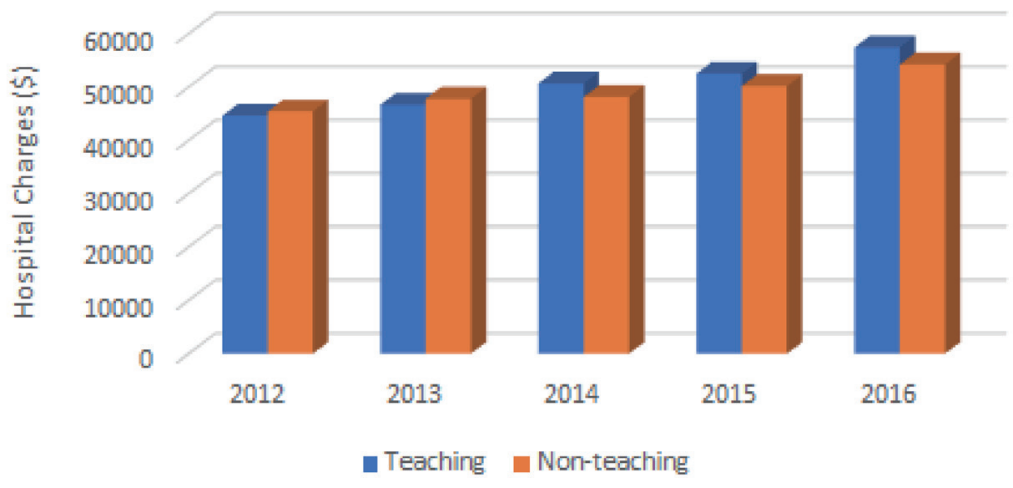

Figure 3. Trend of hospital charges for seizures in teaching vs. non-teaching hospitals in USA from 2012 to 2016. 
in teaching hospitals, teaching hospitals performed more complex procedures and had superior outcomes for these complex surgical procedures compared to non-teaching hospitals [10]. There is also previous evidence to suggest that higher patient and procedural complexity is often noted at teaching hospitals that contribute to the greater complications and morbidity associated with teaching hospitals [10]. It is also been observed that other factors such as age and comorbidities have a much higher impact on mortality than does the teaching status of a hospital $[10,11]$. Our study suggests that in the case of seizures, both teaching and non-teaching hospitals likely practice the same standard of care, and treat similar complexity of seizure caseloads such that there is no observed difference in percentage of in-hospital deaths.

Previous studies have often suggested higher hospital charges among teaching hospitals as compared to non-teaching hospitals. Iezzoni et al particularly observed this difference in tertiary teaching hospitals. They reported that disease-specific severity and the comorbidity index were shown to have a strong linear relationship to DRG-associated costs and accounted for much of the perceived higher costs when comparing tertiary teaching hospitals to non-teaching hospitals. However, such differences were not noted among non-tertiary teaching hospitals [12]. Furthermore, our study did not differentiate between the levels of teaching hospitals and found no difference between hospital charges. This may be due to the limitations of our study that was unable to stratify teaching levels. While studies regarding in-hospital charges of seizures are limited, there appears to be a significant increase in hospital charges in both teaching and non-teaching hospitals in comparing average 2012 to 2016 . These charges are likely due to a combination of advancing technology, previously labeled as the main driver of health care expenditure, as well as general and medical inflation and population growth and demand for hospital resources $[13,14]$. These factors do not differ between teaching and non-teaching hospitals and would be a possible explanation as to why teaching status did not alter hospital charges in our study.

Our study demonstrated that teaching hospitals did have a significantly higher LOS compared to non-teaching hospitals in 2 of the 5 years analyzed. There are conflicting studies on LOS between teaching and non-teaching hospitals, with a sparsity of topics related to epilepsy and seizures. Hayanga et al demonstrated and commented on the longer LOS observed in major teaching hospitals, particularly when examined in the context of specialized procedures [15]. This is possibly due to the fact that teaching hospitals have more complicated patients with comorbidities and moreover, complex systems of care set in place for the higher volume and higher complexity of patients they admit. These systems, though may improve outcomes for complex patients, may also increase LOS in patients overall. Differences in resource allocation and coordination of care may also account for the longer LOS seen in teaching hospitals due to the training physician staff and higher volume of patients [10]. These factors all possibly contribute to the increased LOS we observed in our study. However, further research needs to be conducted to investigate if patient demographics and comorbidities differ between teaching and non-teaching hospitals in the context of those presenting with seizures, and whether these factors impact mortality, LOS, and hospital charges outcomes. Further investigation is needed to stratify patient complexities and hospital characteristics in order to determine if there is ever a need for teaching status to play a role in decision in treatment.

\section{Conclusions}

Our data showed that the teaching and non-teaching hospitals do not have a significant difference in mortality for patients with seizures associated with complication or comorbidity. There might however be an increased LOS in teaching hospitals, but regardless hospital charges remain relatively consistent regardless of the teaching status.

\section{Acknowledgments}

None to declare.

\section{Financial Disclosure}

Authors have no financial disclosure relevant to this article.

\section{Conflict of Interest}

Authors have no conflict of interest relevant to this article.

\section{Informed Consent}

Not applicable.

\section{Author Contributions}

AY: writing the manuscript and entire analysis; AS: mentor and design of the project. VE edited, added and rewrote significant parts of the draft.

\section{Data Availability}

The data supporting the findings of this study are available to public at: https://hcupnet.ahrq.gov/\#setup.

\section{References}

1. Epilepsy. World Health Organization. 2019. Available at: https://www.who.int/news-room/fact-sheets/detail/epilepsy. Accessed on March 25, 2020.

2. Huff JS, Morris DL, Kothari RU, Gibbs MA, Emergency Medicine Seizure Study G. Emergency department management of patients with seizures: a multicenter study. 
Acad Emerg Med. 2001;8(6):622-628.

3. Martindale JL, Goldstein JN, Pallin DJ. Emergency department seizure epidemiology. Emerg Med Clin North Am. 2011;29(1):15-27.

4. Zack MM, Kobau R. National and state estimates of the numbers of adults and children with active epilepsy United States, 2015. MMWR Morb Mortal Wkly Rep. 2017;66(31):821-825.

5. Ottman R, Lipton RB, Ettinger AB, Cramer JA, Reed ML, Morrison A, Wan GJ. Comorbidities of epilepsy: results from the Epilepsy Comorbidities and Health (EPIC) survey. Epilepsia. 2011;52(2):308-315.

6. Burke LG, Frakt AB, Khullar D, Orav EJ, Jha AK. Association between teaching status and mortality in US hospitals. JAMA. 2017;317(20):2105-2113.

7. Ayanian JZ, Weissman JS. Teaching hospitals and quality of care: a review of the literature. Milbank Q. 2002;80(3):569-593, v.

8. Stowers J, Ahmadi N, Seifi A. The trend of in-hospital epilepsy and its mortality in the USA: a national analysis during 1997 - 2014. Journal of Neurology Research. 2020;10:3-6.

9. Hyder O, Sachs T, Ejaz A, Spolverato G, Pawlik TM. Impact of hospital teaching status on length of stay and mortality among patients undergoing complex hepatopan- creaticobiliary surgery in the USA. J Gastrointest Surg. 2013;17(12):2114-2122.

10. Fineberg SJ, Oglesby M, Patel AA, Pelton MA, Singh K. Outcomes of cervical spine surgery in teaching and non-teaching hospitals. Spine (Phila Pa 1976). 2013;38(13):1089-1096.

11. Sandhu A, Moscucci M, Dixon S, Wohns DH, Share D, LaLonde T, Smith D, et al. Differences in the outcome of patients undergoing percutaneous coronary interventions at teaching versus non-teaching hospitals. Am Heart J. 2013;166(3):401-408.

12. Iezzoni LI, Shwartz M, Moskowitz MA, Ash AS, Sawitz E, Burnside S. Illness severity and costs of admissions at teaching and nonteaching hospitals. JAMA. 1990;264(11):1426-1431.

13. Chandra A, Skinner J. Technology growth and expenditure growth in health care. Journal of Economic Literature. 2012;50(3):645-680.

14. Ashby JL, Jr., Lisk CK. Why do hospital costs continue to increase? Health Aff (Millwood). 1992;11(2):134-147.

15. Hayanga AJ, Mukherjee D, Chang D, Kaiser H, Lee T, Gearhart S, Ahuja N, et al. Teaching hospital status and operative mortality in the United States: tipping point in the volume-outcome relationship following colon resections? Arch Surg. 2010;145(4):346-350. 